Katarzyna Buszko*1, Agata Kosobucka*2, Piotr Michalski ${ }^{3}$, Łukasz Pietrzykowski ${ }^{3}$, Aleksandra Jurek ${ }^{4}$, Marzena Wawrzyniak ${ }^{4}$, Karolina Obońska ${ }^{4}$, Mirosława Felsmann², Aldona Kubica ${ }^{3}$

${ }^{1}$ Department of Theoretical Foundations of Biomedical Science, and Medical Informatics, Nicolaus Copernicus University, Collegium Medicum in Bydgoszcz, Poland

${ }^{2}$ Laboratory of Basic Clinical Skills and Medical Simulations, Nicolaus Copernicus University, Collegium Medicum in Bydgoszcz, Poland

${ }^{3}$ Department of Health Promotion, Nicolaus Copernicus University, Collegium Medicum in Bydgoszcz, Poland

${ }^{4}$ Department of Cardiology and Internal Diseases, Nicolaus Copernicus University, Collegium Medicum in Bydgoszcz, Poland

\title{
The readiness for hospital discharge of patients after acute myocardial infarction: a new self-reported questionnaire
}

\author{
Corresponding author: \\ Katarzyna Buszko, PhD \\ Department of Theoretical \\ Foundations of Biomedical Science \\ and Medical Informatics, \\ Nicolaus Copernicus University, \\ Collegium Medicum in Bydgoszcz \\ 13 Jagiellońska St., \\ 85-067 Bydgoszcz, Poland \\ Tel. 525853428 \\ E-mail: buszko@cm.umk.p
}

Medical Research Journal 2017;

Volume 2, Number 1, 20-28

10.5603/MRJ.2017.0004

Copyright (C) 2017 Via Medica

ISSN 2451-2591

\begin{abstract}
Introduction. Medical care providers are responsible for adequate preparation of patients for discharge from the hospital. The purpose of this study was to validate a new self-reported questionnaire assessing the readiness of patients for hospital discharge.

Methods. The Readiness for Hospital Discharge after Myocardial Infarction Scale (RHD MIS) was validated in 201 patients, 57 (29\%) females and 144 (71\%) males (mean age $63.3 \pm 11.3$ ), hospitalised due to myocardial infarction

Results. For the considered 23 items the $\alpha$-Cronbach coefficient was 0.789 , indicating a high level of reliability and homogeneity of the questionnaire. The RHD MIS fulfilled the assumption of factor analysis: the determinant of correlation matrix was 0.001, Kaiser-Mayer-Olkin (K-M-O) statistic was 0.723, and the Bartlett' test of sphericity was statistically significant. The analysis of internal consistency of the three areas confirm the rightness of the distinguishing of three subscales. Answers to each item were assigned a score from 0 to 3. The highest total score is 69 points. The total score of the scale and total scores of the subscales have skewed distributions and statistically significant results of Shapiro-Wilk test $(p<0.001)$. The scoring less than 44 points for the entire questionnaire indicates low readiness, obtaining between 44 and 57 points indicates medium readiness, and scores over 57 points are classified as high readiness for discharge from hospital.

Conclusions. The validation procedure revealed that RHD MIS is a reliable and homogeneous tool to measure the readiness of patients for hospital discharge. The set of items divided into three subscales allows subjective and objective evaluation of the patient's knowledge and expectations. Further investigation is needed to assess the potential impact of RHD MIS scoring on long-term outcome.
\end{abstract}

Key words: readiness for hospital discharge, self-reported questionnaire, scale

Med Res J 2017; 2 (1): 20-28

\section{Introduction}

Despite intense development of invasive treatment techniques and a wide range of available pharmacotherapy, ischaemic heart disease still remains a challenge for a therapeutic team involved in the care of this group of patients [1]. It is estimated that as many as $20 \%$ of patients make major mistakes regarding discharge recommendations within the first three weeks [2]; moreover, $60-80 \%$ of patients do not follow recommendations regarding long-term treatment [3, 4], which results in substantial health consequences. Medical care providers are responsible for adequate preparation of patients for discharge from the hospital [5]. Galvin et al. [6] proposed an operational definition of readiness for hospital discharge that includes following attributes:

*both authors contributed equally and should be considered first authors 
physical stability, adequate support, psychological ability, and adequate information and knowledge. A better understanding of this phenomenon will assist in recognising, measuring, and implementing interventions where necessary, to ensure patients are ready for hospital discharge [6].

Considering these facts, it is justified to create and implement into daily practice a tool assessing the level of readiness for discharge of patients leaving hospital. The tool needs to be simple and reliable, which enables its use in clinical conditions. It should allow a quick and efficient assessment to be made of the patients' knowledge, expectations, and concerns, as well as indicating the field requiring additional intervention.

The purpose of the study was to validate a new self-reported questionnaire assessing the readiness of patients for hospital discharge.

\section{Materials and methods}

\section{Study group}

The study group consisted of 201 patients, 57 (29\%) females and 144 (71\%) males, aged 30-91 years (mean age $63.3 \pm 11.3$ ), with coronary artery disease (CAD), and treated with percutaneous coronary intervention (PCl). The study was conducted between May 2015 and July 2016. The study population characteristics are displayed in Table 1.

Statistical analyses were conducted using IBM SPSS Statistic 23.0. All statistical tests were performed on the significance level $\alpha=0.05$.

The Self-Reported Questionnaire for Patients After Myocardial Infarction Treated with Primary Coronary Intervention is presented in Table 2.

\section{Survey validation}

The validation of the survey was conducted in accordance with the validation procedure. The internal consistency was calculated using the $\alpha$-Cronbach coefficient. Factor analysis was conducted using principal component analysis with Varimax rotation. The Catell criterion and Kaiser criterion were considered in order to determine components of the questionnaire. The factor analysis was preceded by checking: the determinant of correlation matrix, Kaiser-Mayer-Olkin (K-M-O) statistic, and the Bartlett test of sphericity. The scale and subscale levels were determined based on the percentiles scale.

Despite filling the RHD MIS, patients were asked to express their opinions associated with readiness to be discharged from the hospital (Tab. 3).
Table 1. Characteristics of patients

\begin{tabular}{lcc}
\hline Parameter & $\begin{array}{c}\text { Category/ } \\
\text { /value }\end{array}$ & $\begin{array}{c}\text { Number of } \\
\text { patients }\end{array}$ \\
\hline Age & $>65$ & $81(40 \%)$ \\
Education & Primary & $22(11 \%)$ \\
& Vocational & $68(33.8 \%)$ \\
& Secondary & $80(39.8 \%)$ \\
Employment status & Higher & $31(15.4 \%)$ \\
& Employed & $87(43.3 \%)$ \\
& Unemployed & $9(4.4 \%)$ \\
Marital status & Pensioner & $81(40.3 \%)$ \\
& Retiree & $24(12 \%)$ \\
Previous hospitalisation & Unmarried & $12(6 \%)$ \\
for CAD & Married & $176(87.5 \%)$ \\
Previous diagnosis & Widowed & $13(6.5 \%)$ \\
& Yes & $73(36.3 \%)$ \\
& Myocardial infarction & $55(27.4 \%)$ \\
& Hypertension & $124(61.8 \%)$ \\
Smoking (currently) & Hyperlipidaemia & $102(50.8 \%)$ \\
Previous treatment & Diabetes & $52(25.9 \%)$ \\
& Yes & $70(34.8 \%)$ \\
& PCl & $73(36.3 \%)$ \\
\hline & CABG & $24(12 \%)$ \\
\hline
\end{tabular}

$\mathrm{PCl}$ - percutaneous coronary intervention; $\mathrm{CABG}$ - coronary artery bypass grafting

\section{Results}

\section{Validation of the RHD MIS}

The questionnaires were anonymously completed by 201 patients. The questionnaire consisted of 23 questions, for which there were four for possible answers to choose from. The answers are scored from 0 to 3 points. In the case of items: A, B, C, D, and $E$ the answers are not scored. They are connected with the assessment of the patient's situation, and do not measure intensity of any feature. Therefore, this set of items was not included in the validation procedure. In this procedure we analysed 18 statements self-reported by the patients in response to the following items: 1.1-1.7 and 3.1-3.9, and seven items assessed by the medical staff during a consultation with the patient: 2.1-2.7. All statistical tests were performed on the significance level $\alpha=0.05$. The Shapiro-Wilk test was used in the assessment of the distribution of the total score and the scores of each item. In each case the test was statistically significant $(p<0.001)$. The distributions of scores for particular items were usually skewed; in particular, the total score had left-skewed distribution with kurtosis -0.85 and skew -0.29 . 
Table 2. The Self-Reported Questionnaire for Patients After Myocardial Infarction Treated with Primary Coronary Intervention

\section{The Readiness for Hospital Discharge \\ After Myocardial Infarction Scale (RHD MIS) \\ Author: Aldona Kubica}

1. Knowledge - the subjective assessment (Mark one correct answer)

1.1. Do you know the reason for your hospitalisation?

a. Yes.

b. I guess so.

c. I'm not sure.

d. I do not.

1.2. Did you understand your doctor's recommendations regarding diet?

a. Yes.

b. I guess so.

c. I'm not sure.

d. I do not.

1.3. Did you understand your doctor's recommendations regarding physical activity?

a. Yes.

b. I guess so.

c. I'm not sure.

d. I do not.

1.4. Did you understand your doctor's recommendations regarding medication?

a. Yes.

b. I guess so.

c. I'm not sure.

d. I do not.

1.5. Did you understand your doctor's recommendations regarding follow-up visits?

a. Yes.

b. I guess so.

c. I'm not sure.

d. I do not.

1.6. Do you know the symptoms that should cause a call for ambulance?

a. Yes.

b. I guess so.

c. I'm not sure.

d. I do not.

1.7. Do you know the symptoms that should result in an additional visit to the doctor?

a. Yes.

b. I guess so.

c. I'm not sure.

d. I do not.

\section{Knowledge - objective assessment}

2.1. What was the reason of your hospitalisation? (Mark one correct answer)
a. Myocardial infarction.
b. Coronary heart disease.
c. Heart disease.
d. Other illness.

2.2. Recommendations regarding patient's diet (Mark all the appropriate answers)

a. Limiting fatty meat.

b. Lean meat is allowed.

c. Increase the consumption of raw fruits.

d. Limiting yellow cheeses.

e. Skimmed milk is allowed.

$\mathrm{f}$. Increase the consumption of raw vegetables. g. Limit high-cholesterol products such as egg yolks.

h. Small amounts of red wine are allowed.

i. Increase the consumption of marine fish.

2.3. Recommendations regarding patient's physical activity (Mark all the appropriate answers)

a. Systematic physical exercise is beneficial - at least 3-4 times a week.

b. Moderate physical activity is preferred for about 30 minutes daily.

c. In case of discomfort, stop the effort.

d. Movement effort is preferred, e.g. walking, running, cycling, swimming.

2.4. Recommendations regarding patient's medication (Mark all the appropriate answers)

a. All medicines should be taken systematically.

b. Do not discontinue medicines without consulting your doctor.

c. Contact your doctor if you experience side effects.

2.5. Recommendations regarding patient's follow-up visits (Mark all the appropriate answers)

a. Follow-up visits should be executed regardless of being.

b. In a case of recurrence of illness symptoms, you should apply for an additional visit.

c. In a case of any new symptoms, you should apply for an additional visit.

2.6. Symptoms that should cause a call for ambulance (Mark all the appropriate answers)

a. Chest pain at rest.

b. Dyspnoea at rest.

c. Fainting or loss of consciousness.

2.7. Symptoms that should cause an extra visit to the doctor (Mark all the appropriate answers)

a. Exertional chest pain.

b. Exercise breathlessness.

c. Dizziness.

d. Heart palpitations.

e. Elevated blood pressure.

f. Swelling of the legs.

g. Other symptoms associated with medication.

\section{Expectations (Mark one correct answer)}

3.1. Do you need additional information regarding the disease?

a. Yes.

b. I guess so.

c. I'm not sure.

d. I do not.

3.2. Do you need additional information regarding applied medication?

a. Yes.

b. I guess so.

c. I'm not sure.

d. I do not.

3.3. Do you need additional information regarding diet?

a. Yes.

b. I guess so.

c. I'm not sure.

d. I do not.

3.4. Do you need additional information regarding physical activity?

a. Yes.

b. I guess so.

c. I'm not sure.

d. I do not. 
Table 2 (cont.). The Self-Reported Questionnaire for Patients After Myocardial Infarction Treated with Primary Coronary Intervention cont

3.5. Do you need additional information regarding medication after discharge from the hospital?
a. Yes.
b. I guess so.
c. I'm not sure.
d. I do not.

3.6. Do you need additional information regarding prevention of the illness recurrence?
a. Yes.
b. I guess so.
c. I'm not sure.
d. I do not.

3.7. Do you need additional information regarding follow-up visits?
a. Yes.
b. I guess so.
c. I'm not sure.
d. I do not.

3.8. Do you need additional information regarding symptoms of the disease?

a. Yes.

b. I guess so.

c. I'm not sure.

d. I do not.

3.9. Do you need any other additional information?
a. Yes.
b. I guess so.
c. I'm not sure.
d. I do not.

The basic tool used to check the internal consistency of the questionnaire was the $\alpha$-Cronbach coefficient. For the considered 23 items the $\alpha$-Cronbach coefficient was 0.789 , which indicates a high level of reliability and homogeneity of the questionnaire. We also calculated the $\alpha$-Cronbach coefficient for the questionnaire after removing individual questions. The $\alpha$-Cronbach coefficient was slightly higher when items: $1.1(\alpha$-Cronbach $=0.793)$, $1.2(\alpha$-Cronbach $=0.792), 1.3(\alpha$-Cronbach $=0.79)$ and $2.1(\alpha$-Cronbach $=0.79)$ were not used for computation. Due to the high value of the $\alpha$-Cronbach coefficient we decided not to remove the items from the questionnaire.

The next step in the validation procedure is the factor analysis [7]. However, in the case of such a high value of the $\alpha$-Cronbach coefficient $(0.789)$ the factor analysis is not necessary because it is obvious that only one component will be loaded by the majority of items. We performed the factor analysis. The set of 23 items fulfilled the following assumptions of factor analysis: the determinant of the correlation matrix was 0.001 , Kaiser-Mayer-Olkin (K-M-O) statistic was 0.723 , and the Bartlett' test of sphericity was statistically significant. The factor analysis was conducted using principal component analysis with Varimax rotation. We determined the components of the questionnaire firstly in line with the Kasier criterion and we obtained
Table 3. Patients opinions associated with readiness to be discharged from the hospital

OPINIONS (After each question select one answer that seems to be the most appropriate)

A. Do you think that the illness being the reason for your hospitalisation is serious?

a. Yes.

b. I guess so.

c. I'm not sure.

d. I do not.

B. Do you think that despite the medication, you need to change your lifestyle to prevent illness recurrence?

a. Yes.

b. I guess so.

c. I'm not sure.

d. I do not.

C. Do you think that systematic medication reduces the risk of reinfarction?

a. Yes.

b. I guess so.

c. I'm not sure.

d. I do not.

D. Can you rely on the help of family or other people to comply with your doctor's recommendations?

a. Yes.

b. I guess so.

c. I'm not sure.

d. I do not.

E. Do you think your return home is associated with additional hazards?

a. Yes.

b. I guess so.

c. I'm not sure.

d. I do not.

Table 4. The scale's levels of the readiness of patients for hospital discharge

\begin{tabular}{lcc}
\hline Percentile & Total score & Readiness \\
\hline $30-70$ & $44-57$ & Medium level \\
& $\begin{array}{c}\text { Subjective assessment of the } \\
\text { patient's knowledge }\end{array}$ & \\
$30-50$ & $16-18$ & Medium level \\
& $\begin{array}{c}\text { Objective assessment of the } \\
\text { patient's knowledge }\end{array}$ & \\
$30-70$ & $13-18$ & Medium level \\
& The patient's expectations & \\
$30-70$ & $14-22$ & Medium level \\
\hline
\end{tabular}

seven components, but according to Catell criterion (based on the screen plot presented in Figure 1) we could choose only one component $[7,8]$. The analysis of factor loadings showed that in the majority of items only one component was loaded.

The described results prompted us to divide the questionnaire based on the merits and experience of the questionnaire's author. We divided the 23 items into three fields: 


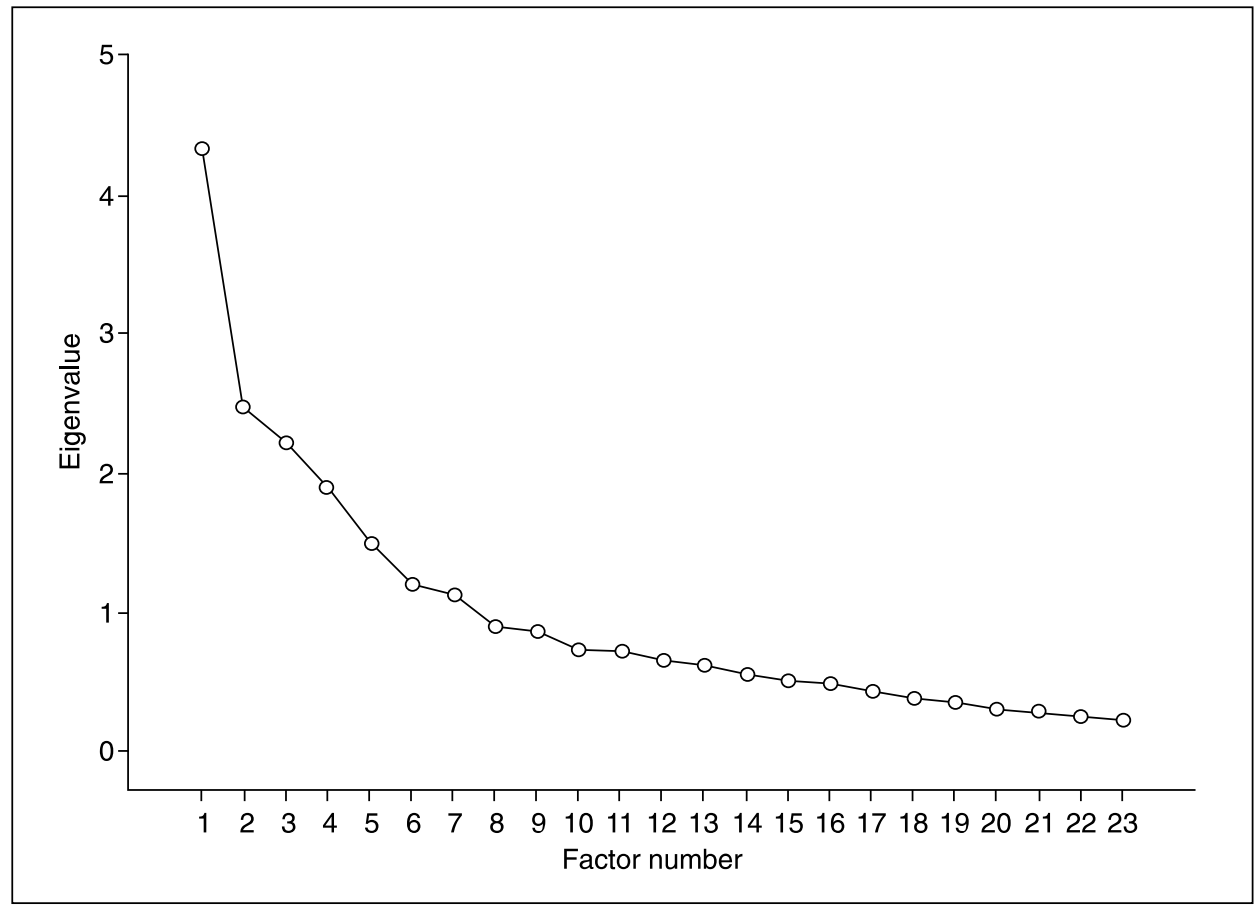

Figure 1. Scree plot

- the subjective assessment of the patient's knowledge about the illnesses and treatment; items 1.1-1.7;

- the subjective assessment of the patient's knowledge about the illnesses and treatment; items 2.1-2.7;

- the patient's expectations; items 3.1-3.9.

The investigation of the internal consistency of the first area showed that it has an adequate level of reliability and homogeneity with the $\alpha$-Cronbach coefficient at 0.568 . After removing individual questions the $\alpha$-Cronbach was slightly higher (when items 1.1, 1.2 and 1.7 were removed).

The calculation of the $\alpha$-Cronbach coefficient for the second area indicated good reliability and homogeneity $(\alpha$-Cronbach coefficient $=0.662)$. Recalculation of the $\alpha$-Cronbach coefficient after removing items 2.1 and 2.7 showed that the coefficient would be only slightly higher.

The $\alpha$-Cronbach coefficient for the third area was 0.798 , which indicates high reliability and homogeneity of the set of items. Removing any items increased the $\alpha$-Cronbach coefficient. The analysis of the internal consistency of the three areas confirmed the suitability of distinguishing the three subscales. In the analysed questionnaire one would assess three fields of the readiness of patients for hospital discharge.

\section{Interpretation of RHDS results}

The described questionnaire should be associated with a scale that indicates the level of the measured effect. As we mentioned previously the answers to each item were assigned a score from 0 to 3 . The highest total score is 69 points. In the case of a subscale for the component of the patient's knowledge about his/her subjective assessment the highest total score was 21 points, and for the component of the patient's knowledge in an objective assessment it was also 21 points. The total score for the subscale of expectations was 27 points. The total score of the scale and total scores of the subscales had skewed distributions (Fig. 1-4) and statistically significant results of Shapiro-Wilk test $(p<0.001)$. Therefore, we determined the scale levels based on a percentile scale. The percentiles determined for the scale and for the subscales are presented in Table 4.

In case of the total score, the second, and third subscales, we assumed that a score less than the $30^{\text {th }}$ percentile indicated low readiness, a score between the $30^{\text {th }}$ and $70^{\text {th }}$ percentile indicated medium readiness, and a score higher than the $70^{\text {th }}$ percentile indicated high readiness. In the case of first subscale a score higher than the $30^{\text {th }}$ percentile indicated low readiness, a score between the $30^{\text {th }}$ and $50^{\text {th }}$ percentile indicated medium readiness, and a score higher than the $50^{\text {th }}$ percentile indicated high readiness (Tab. 4). This means that scoring less than 44 points for the entire questionnaire indicated low readiness, obtaining between 44 and 57 points indicated medium readiness, and scores over 57 points were classified as high readiness. In the case of the first subscale for 


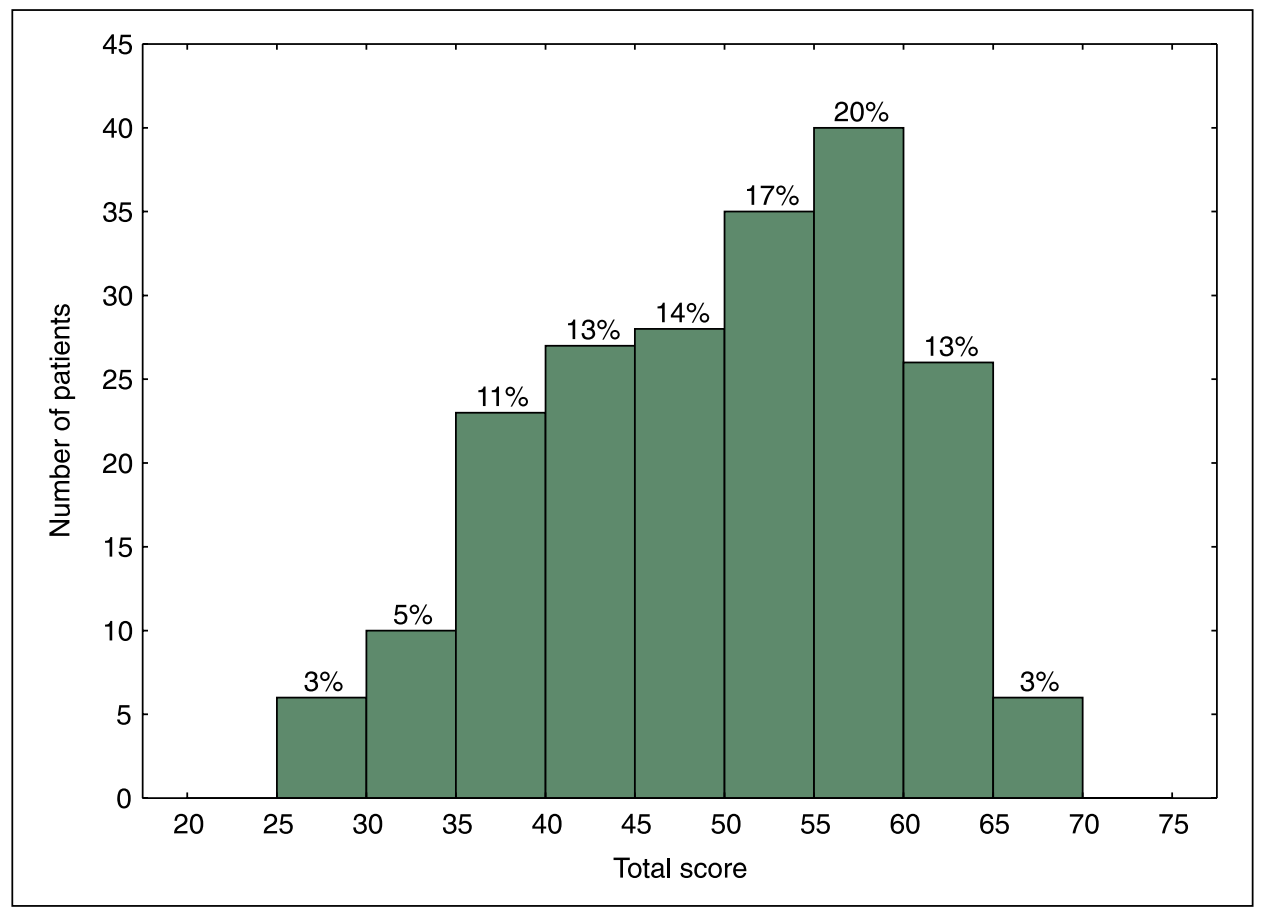

Figure 2. The distribution of the total score of the readiness of patients for hospital discharge

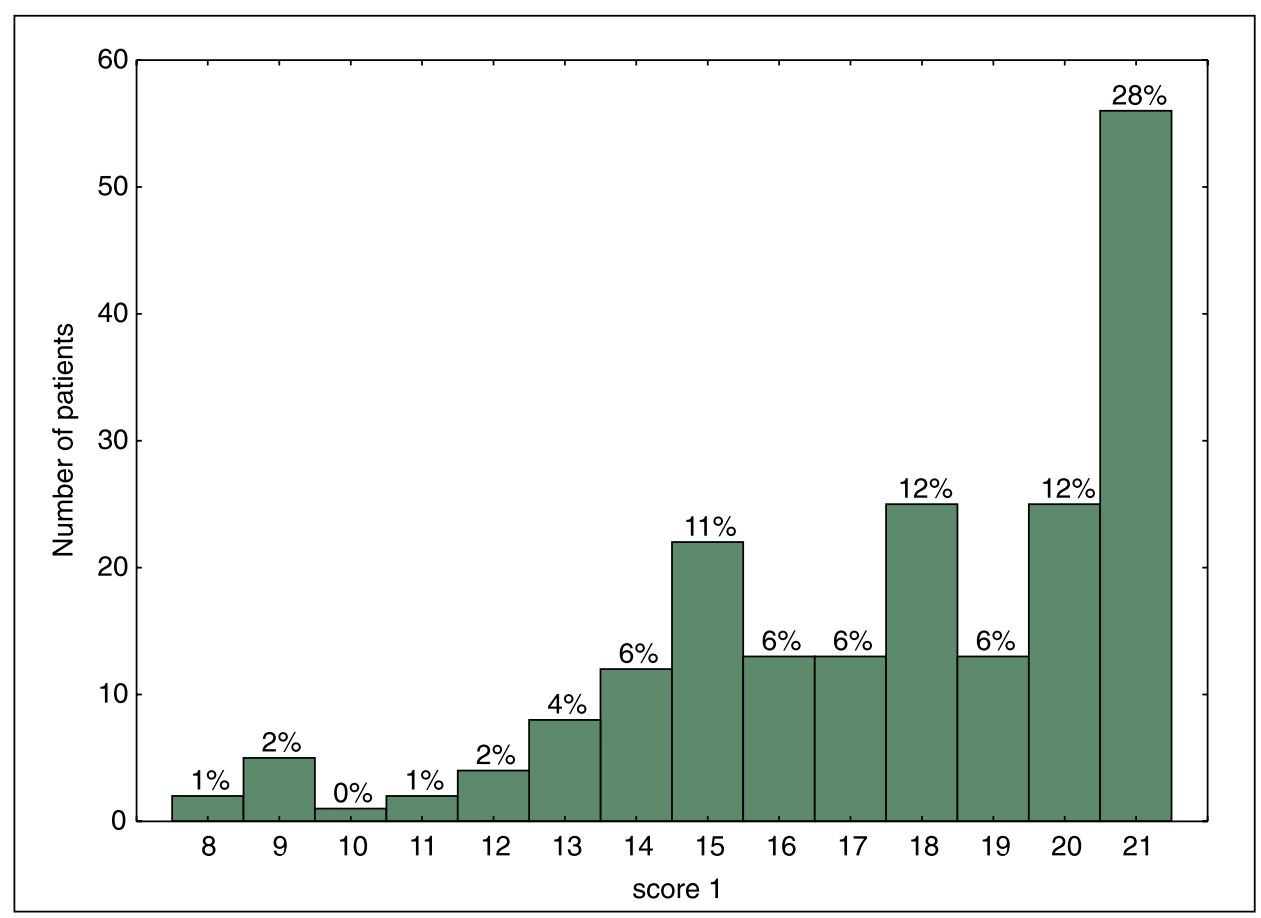

Figure 3. The distribution of the score in the area of the subjective assessment of the patient's knowledge

the subjective assessment of the patient's knowledge, obtaining under 16 points indicated a low level, scores between 16 and 18 were allocated to the medium level, and scores over 18 points were classified as the high level (Tab. 4). In the second subscale for the objective assessment of the patient's knowledge a score under 13 points indicated low readiness, a score between 13 and 18 corresponded to the medium level, and a score over 18 points indicated a high result (Tab. 4). The levels of the patient's readiness for the third sub- 


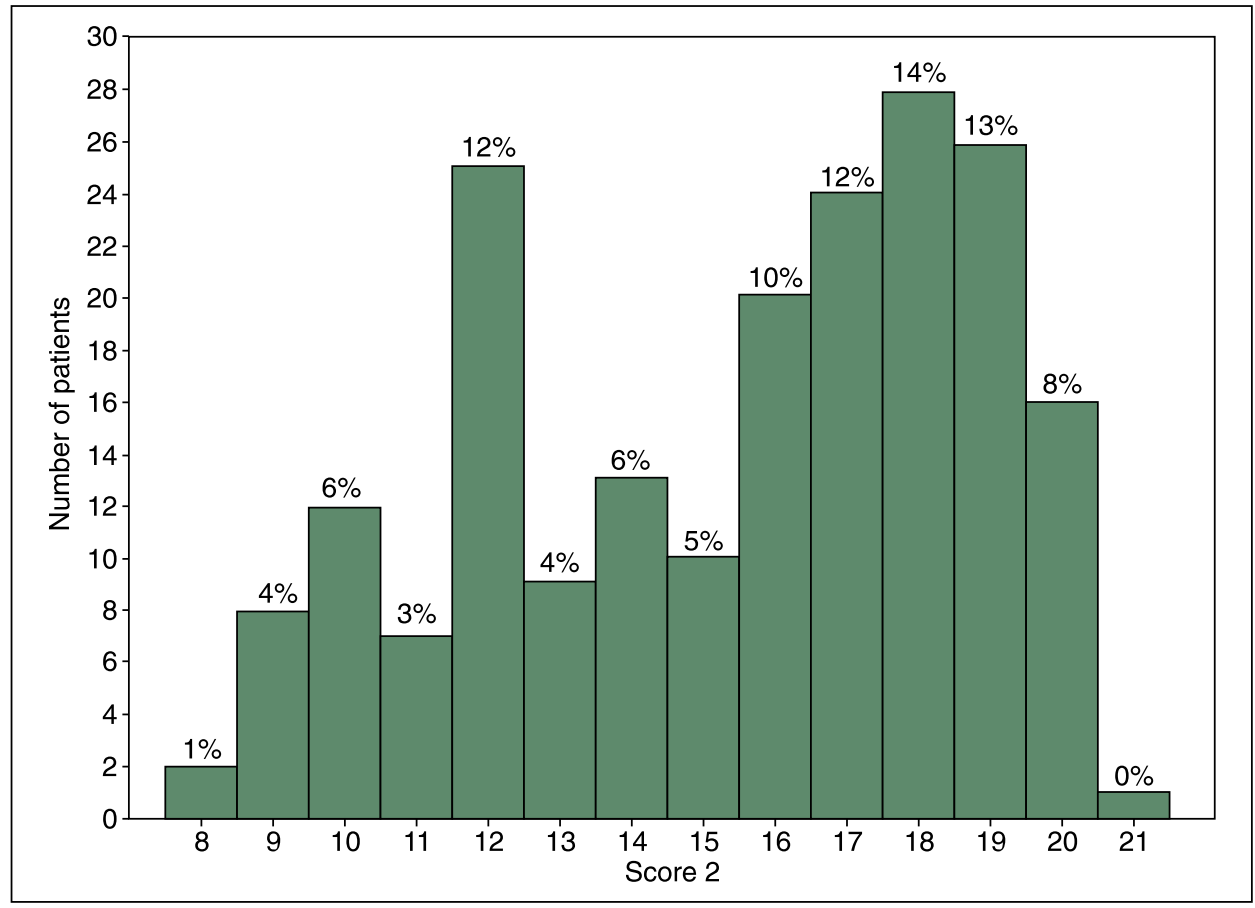

Figure 4. The distribution of the score in the area of the objective assessment of patient's knowledge

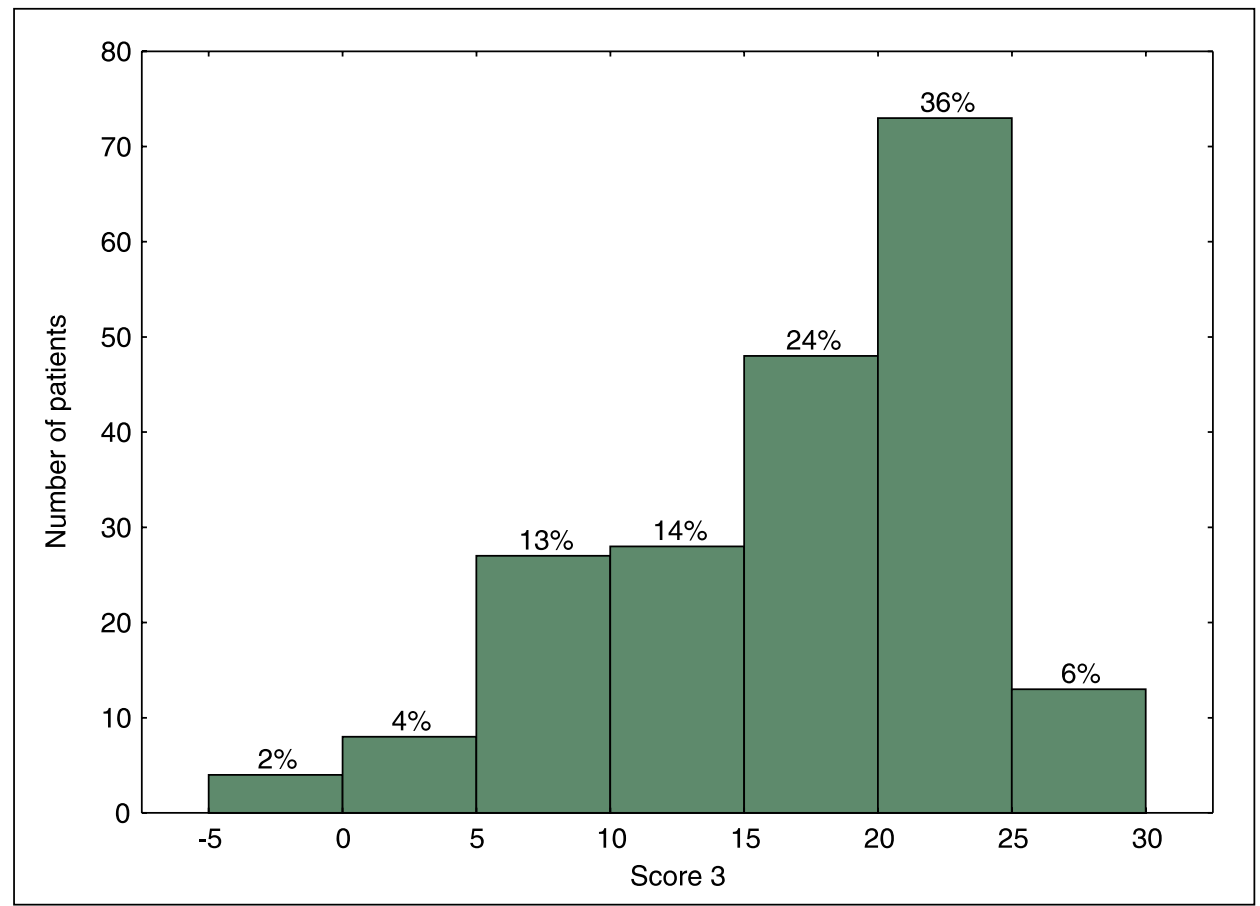

Figure 5. The distribution of the score in the area of the patient's expectations

scale were as follows: low level under 14 points, medium level between 14 and 22 points, and high level over 22 points. The distribution of total score and the score of the areas of the readiness of patients for hospital discharge are presented in Figure 2-5.

\section{Discussion}

The length of in-hospital stay systematically declines, resulting with time shortening for education of patients and their families with regard to post-discharge 
treatment [9]. Medical care providers are perceived to be responsible for providing reliable information concerning treatment after hospital discharge [5]. However, the study conducted among older patients discharged from an emergency department in Finland showed that only one in four patients and $40 \%$ of family members received no discharge education [10]. Nevertheless, discharge education was associated with a higher level of discharge readiness, both among patients and family members [10]. In a prospective observational study conducted in 15 hospitals in four European countries, a readmitted patient reported not feeling ready for discharge at index admission. The authors concluded that healthcare workers should question patients' readiness to go home [11]. For quality improvement of the hospital discharge procedure as well as for research purposes, clinicians need appropriate, reliable, and valid survey instruments to measure the patients' readiness for discharge. The discharge procedure is a multifactorial, interdisciplinary, individualised process of transition from hospital to outpatient care, requiring efforts aimed to meet patients' expectations and needs as well as to negotiate the agreement regarding a therapeutic plan for the post-discharge period. Such an approach should improve patients' satisfaction and adherence to treatment. Therefore, we have developed a new scale dedicated specifically to patients hospitalised due to myocardial infarction. The RHD MIS combines patients' and medical professionals' perspectives regarding readiness for discharge from the hospital, applying integrated subjective and objective assessment. According to our knowledge, it is the first validated survey taking into account specificity of patients with MI. Until now, the dedicated questionnaire evaluating readiness for hospital discharge was developed only for patients with schizophrenia [12].

The RHD MIS was shown to have a high level of reliability and the homogeneity of the $\alpha$-Cronbach coefficient was 0.789 for 23 items. The $\alpha$-Cronbach coefficient only slightly improved after removal of individual questions, so we decided not to remove any items from the questionnaire.

Developed in the USA by Weiss, the Readiness for Hospital Discharge Scale (RHDS), probably the most widely used instrument measuring an individual's self-perception of readiness before leaving the hospital, is characterised by very good psychometric properties with an $\alpha$-Cronbach of 0.90 for the final 21 -item version of the scale [13]. The reliability of the Chinese and French versions were also high, with $\alpha$-Cronbach coefficients of 0.89 and 0.80 , respectively $[14,15]$. The internal consistency of another instrument to measure preparedness for hospital discharge: the Brief PREPARED (B-PREPARED) reported by Graumlich et al. [16], was also acceptable ( $\alpha$-Cronbach of 0.76$)$.
The RHD MIS analysis of factor loadings showed that in the majority of items only one component is loaded. Therefore, the questionnaire was divided, based on the merits and experience of the questionnaire's author, into three fields.

The analysis of internal consistency of the three areas confirmed the rightness of distinguishing the subscales. Each RHD MIS item was assigned a score from 0 to 3 . The highest total score is 69 points with 21 for each of the first and second subscales and 27 points for the third subscale. The RHD MIS scoring over 57 points for the entire questionnaire indicates high readiness for discharge, while less than 44 points suggests low readiness, and a score of between 44 and 57 points is classified as medium readiness.

Assessing patient readiness for hospital discharge before leaving hospital could improve the planning of the discharge process, including additional education to achieve better patient preparedness and care coordination [15]. Effective education should have a favourable influence on patients' behaviour concerning risk factor reduction and medication [17]. Schmocker et al. [18] evaluated the association of readiness for discharge with patient satisfaction and readmission. Using the survey question "Did you feel ready for discharge?", patients were divided into two groups according to degrees of readiness. Those defined as ready for discharge had higher overall hospital satisfaction, higher physician communication scores, and higher nursing communication scores. Readmission rates, however, were similar between the groups [18]. Weiss et al. [19] validated a short, eight-item version of RHDS for discharge readiness assessment, its associations with 30-day readmissions, and emergency department visit rates. Nurses and patients independently completed the questionnaire on the day of discharge. Nurse judgement of low discharge readiness was associated with a six- to nine-fold increase in readmission risk; however, patient self-assessment was associated neither with readmission nor with visits in emergency department [19]. Patient self-assessment is a purely subjective assessment, while nurse evaluation is more objective $[20,21]$. Such an approach was applied in RHD MIS in the first and second subscale.

Implementation of a discharge readiness report into the electronic health record may further improve the efficiency and effectiveness of care transitions by allowing for proactive discharge planning and improved interdisciplinary communication [22].

\section{Limitations of the study}

The study was conducted in a single university hospital, and the sample characteristics may not reflect the 
types of patients assigned to other cardiology departments. Further application of RHD MIS in multicentre studies will provide an opportunity to characterise this tool in specific subsets of patients.

\section{Conclusions}

The validation procedure revealed that RHD MIS is a reliable and homogeneous tool to measure the readiness of patients for hospital discharge. The set of items divided into three subscales allows subjective and objective evaluation of the patient's knowledge and expectations. Further investigation is needed to assess the potential impact of RHD MIS scoring on long-term outcome.

\section{Conflict of interest: none declared}

\section{References}

1. World Health Organization. Mortality and global burden of disease. http://www.who.int/gho/mortality burden disease/en/.

2. Forster AJ, Murff HJ, Peterson JF, et al. The incidence and severity of adverse events affecting patients after discharge from the hospital. Ann Intern Med. 2003; 138(3): 161-167, indexed in Pubmed: 12558354.

3. Desai NR, Choudhry NK. Impediments to adherence to post myocardial infarction medications. Curr Cardiol Rep. 2013; 15(1): 322 doi: 10.1007/s11886-012-0322-6, indexed in Pubmed: 23250659.

4. Osterberg L, Blaschke T. Adherence to medication. $\mathrm{N}$ Engl J Med. 2005; 353(5): 487-497, doi: 10.1056/NEJMra050100, indexed in Pubmed: 16079372.

5. Visser A, Deccache A, Bensing J. Patient education in Europe: united differences. Patient Educ Couns. 2001; 44(1): 1-5, indexed in Pubmed: 11390150.

6. Galvin EC, Wills T, Coffey A. Readiness for hospital discharge A concept analysis. J Adv Nurs. 2017 [Epub ahead of print] doi: 10.1111/jan.13324, indexed in Pubmed: 28440958

7. Costello AB, Osborne JW. Best practices in exploratory factor analysis: Four recommendations for getting the most from your analysis. Practical Assessment, Research \& Evaluation. 2005; 10(7): $1531-7714$.
8. Rowe P. Essential Statistics for the Pharmaceutical Sciences. John Wiley \& Sons, Chichester 2016: UK

9. Titler MG, Pettit DM. Discharge readiness assessment. J Cardiovasc Nurs. 1995; 9(4): 64-74, indexed in Pubmed: 7666069.

10. Palonen $\mathrm{M}$, Kaunonen $\mathrm{M}$, Helminen $\mathrm{M}$, et al. Discharge education for older people and family members in emergency department: A cross-sectional study. Int Emerg Nurs. 2015; 23(4): 306-311, doi: 10.1016/i.ienj.2015.02.003, indexed in Pubmed: 25770988

11. van Galen LS, Brabrand M, Cooksley T, et al. Patients' and providers' perceptions of the preventability of hospital readmission: a prospective, observational study in four European countries. BMJ Qual Saf. 2017 [Epub ahead of print], doi: 10.1136/bmjgs-2017-006645, indexed in Pubmed: 28642333.

12. Potkin S, Gharabawi G, Greenspan A, et al. Psychometric evaluation of the Readiness for Discharge Questionnaire. Schizophrenia Research. 2005; 80(2-3): 203-212, doi: 10.1016/j.schres.2005.06.021.

13. Weiss ME, Piacentine LB. Psychometric properties of the Readiness for Hospital Discharge Scale. J Nurs Meas. 2006; 14(3): 163-180, indexed in Pubmed: 17278337.

14. Lin $\mathrm{YH}, \mathrm{Kao} \mathrm{CC}$, Huang AM, et al. Psychometric testing of the chinese version of the readiness for hospital discharge scale. Hu Li Za Zhi. 2014; 61(4): 56-65, indexed in Pubmed: 25116315.

15. Mabire C, Lecerf T, Büla $C$, et al. Translation and psychometric evaluation of a French version of the Readiness for Hospital Discharge Scale. J Clin Nurs. 2015; 24(19-20): 2983-2992, doi: 10.1111/jocn.12937, indexed in Pubmed: 26216599

16. Graumlich JF, Novotny NL, Aldag JC. Brief scale measuring patient preparedness for hospital discharge to home: Psychometric properties. J Hosp Med. 2008; 3(6): 446-454, doi: 10.1002/jhm.316, indexed in Pubmed: 19084894

17. Jackevicius CA, Li P, Tu JV. Prevalence, predictors, and outcomes of primary nonadherence after acute myocardial infarction. Circulation. 2008; 117(8): 1028-1036, doi: 10.1161/CIRCULATIONAHA.107.706820, indexed in Pubmed: 18299512.

18. Schmocker RK, Holden SE, Vang X, et al. Association of Patient-Reported Readiness for Discharge and Hospital Consumer Assessment of Health Care Providers and Systems Patient Satisfaction Scores: A Retrospective Analysis. J Am Coll Surg. 2015; 221(6): 1073-10782.e1, doi: 10.1016/j.jamcollsurg.2015.09.009, indexed in Pubmed: 26474513.

19. Weiss ME, Costa LL, Yakusheva O, et al. Validation of patient and nurse short forms of the Readiness for Hospital Discharge Scale and their relationship to return to the hospital. Health Serv Res. 2014; 49(1): 304-317, doi: 10.1111/1475-6773.12092, indexed in Pubmed: 23855675.

20. Kubica A, Obońska K, Fabiszak T, et al. Adherence to antiplatelet treatment with P2Y12 receptor inhibitors. Is there anything we can do to improve it? A systematic review of randomized trials. Curr Med Res Opin. 2016; 32(8): 1441-1451, doi: 10.1080/03007995.2016.118290, indexed in Pubmed: 27112628

21. Kubica A, Obońska K, Kasprzak M, et al. Prediction of high risk of non-adherence to antiplatelet treatment. Kardiol Pol. 2016; 74(1): 61-67, doi: 10.5603/KP.a2015.0117, indexed in Pubmed: 26101025.

22. Tyler A, Boyer A, Martin S, et al. Development of a discharge readiness report within the electronic health record-A discharge planning tool. J Hosp Med. 2014; 9(8): 533-539, doi: 10.1002/jhm.2212, indexed in Pubmed: 24825848 